\title{
HQET Flavor Currents Using Automated Lattice Perturbation Theory
}

\section{Dirk Hesse*}

Universitá degli studi di Parma, Viale G.P. Usberti 7/a, 43100 Parma, Italy

NIC, DESY, Platanenallee 6, 15738 Zeuthen, Germany

E-mail: dirk.hesse@fis.unipr.it

\begin{abstract}
Matrix elements of heavy-light flavor currents play an important role in modern particle physics and precise theory predictions are of interest for phenomenology. Heavy Quark Effective Theory (HQET) is a valuable tool to obtain such predictions. In the HQET matching program of the ALPHA collaboration presently only the temporal component of the axial vector current is included. Extending the matching to the temporal component of the vector current and the spatial components of the axial vector current thus seems desirable. Here we present a recent one-loop study in lattice perturbation theory to test two candidate matching observables for these currents for their quality to guide future non-perturbative investigations [1].
\end{abstract}

PACS: $12.38 . \mathrm{Bx} ; 12.38 . \mathrm{GC} ; 12.39 . \mathrm{Hg} ; 13.20 . \mathrm{He}$

The 30 International Symposium on Lattice Field Theory - Lattice 2012,

June 24-29, 2012

Cairns, Australia

${ }^{*}$ Speaker. 


\section{Introduction}

Matrix elements of heavy-light flavor currents are important theoretical input to constrain physics of and beyond the standard model through decays of heavy mesons. A theoretically clean way to treat systems with one single heavy quark is Heavy Quark Effective Theory (see [2] and references therein), short HQET. From the theory point of view, especially leptonic decays of heavy mesons are relatively easy to treat. Hence next to the parameters of the action, the HQET program of the ALPHA collaboration [3-8] included the temporal component of the heavy-light axial vector current form the very beginning.

Because of the current big interest in flavor physics within the community, it is a logical next step to include all components of the vector and axial vector current to make theory predictions for matrix elements such as for example the one entering in the exclusive semi-leptonic decay $B \rightarrow \pi l v$.

\subsection{HQET and matching}

HQET is a systematic expansion of QCD with one heavy quark in inverse powers of its mass. In addition to the parameters in the HQET action, one has to take care of the coefficients of the currents under investigation as well. The inclusion of all components of the vector and axial vector current increases the numbers of HQET parameters to be fixed at order $1 / m$ to a total of 19 . Considering this rather big number of matching conditions, it seems useful to test them first in relatively cheap perturbative calculations. This possibility is even more attractive since we have the pastor software package [9] for automated lattice perturbation theory calculations at our disposal. Even though perturbative one-loop calculations performed with pastor only require a moderate effort we do not intend to perform the matching of all 19 parameter here. Instead we will focus on the renormalization constants of the temporal component of the vector and the spatial components of the axial vector current. These play an important role since as stated above they were not included in the matching by the ALPHA collaboration so far and enter already at the lowest order in HQET.

The matching procedure consists basically of evaluating the same number of observables $\Phi_{i}$ as the number of parameters to be fixed - in QCD and HQET - and setting $\Phi_{i}^{\mathrm{HQET}}=\Phi_{i}^{\mathrm{QCD}}$ to determine (in fact define) the HQET parameters. The main objective of this work will be to investigate possible matching conditions for the currents mentioned above and estimate the size of higher order contributions in the $1 / m$-expansion. For a precise determination of the parameters, one certainly wants these higher order effects to play as little a role as possible.

\section{Matching of flavor currents}

We work in the Schrödinger functional $[10,11]$ with two mass-less quark flavors, $m_{1}=m_{2}=0$, and a third heavy one whose renormalized mass is as usual given by the dimension-less parameter $z=\bar{m}_{3} L=\bar{m} L$. The boundary fields are given by $\zeta_{i}, \bar{\zeta}_{i}$ and the phase angle for all three flavors is equal and denoted $\theta$. We are interested in the temporal component of the vector current and the spatial components of the axial vector current of a heavy and a light quark,

$$
A_{k}(x)=\bar{\psi}_{3}(x) \gamma_{k} \gamma_{5} \psi_{2}(x), \quad V_{0}(x)=\bar{\psi}_{3}(x) \gamma_{0} \psi_{2}(x),
$$


or to be more precise their matrix elements between heavy-light pseudoscalar states. To this end, we define in a first step the three-point functions (with implicit improvements $b_{\mathrm{A}}, b_{\mathrm{V}}$ [12])

$$
\begin{aligned}
& f_{1}^{\mathrm{A}_{1}}\left(x_{0} ; \theta, z\right)=-\frac{a^{3}}{2} \sum_{\mathbf{x}}\left\langle\bar{\zeta}_{1} \gamma_{5} \zeta_{3} A_{0}(x) \bar{\zeta}_{2}^{\prime} \gamma_{1} \zeta_{1}^{\prime}\right\rangle, \quad \zeta_{i}=\frac{a^{3}}{L^{-3 / 2}} \sum_{\mathbf{x}} \zeta_{i}(\mathbf{x}), \quad \bar{\zeta}_{i}=\frac{a^{3}}{L^{-3 / 2}} \sum_{\mathbf{x}} \bar{\zeta}_{i}(\mathbf{x}), \\
& f_{1}^{\mathrm{V}_{0}}\left(x_{0} ; \theta, z\right)=-\frac{a^{3}}{2} \sum_{\mathbf{x}}\left\langle\bar{\zeta}_{1} \gamma_{5} \zeta_{3} A_{0}(x) \bar{\zeta}_{2}^{\prime} \gamma_{5} \zeta_{1}^{\prime}\right\rangle .
\end{aligned}
$$

To define observables that are suitable to extract the desired matrix elements [13], we form the ratios

$$
\begin{gathered}
\Phi^{\mathrm{V}_{0}}(L, \bar{m})=\log \left(-\frac{Z_{\mathrm{V}} f_{1}^{\mathrm{V}_{0}}(T / 2 ; \theta, z)}{\left[f_{1}(\theta, z) f_{1}(\theta, 0)\right]^{1 / 2}}\right), \quad \Phi^{\mathrm{A}_{k}}(L, \bar{m})=\log \left(-\frac{Z_{\mathrm{A}} f_{1}^{\mathrm{A}_{1}}(T / 2 ; \theta, z)}{\left[f_{1}(\theta, z) k_{1}(\theta, 0)\right]^{1 / 2}}\right), \\
f_{1}(\theta, z)=-\frac{a^{3}}{2} \sum_{\mathbf{x}}\left\langle\bar{\zeta}_{1} \gamma_{5} \zeta_{3} \bar{\zeta}_{3}^{\prime} \gamma_{5} \zeta_{1}^{\prime}\right\rangle, \quad k_{1}(\theta, z)=-\frac{a^{3}}{2} \sum_{\mathbf{x}}\left\langle\bar{\zeta}_{1} \gamma_{1} \zeta_{3} \bar{\zeta}_{3}^{\prime} \gamma_{1} \zeta_{1}^{\prime}\right\rangle .
\end{gathered}
$$

The renormalization constants $Z_{\mathrm{V}}, Z_{\mathrm{A}}$ are fixed by Ward identities [14,15]. The quark masses are renormalized using the lattice minimal subtraction scheme $[16,17]$ at one-loop order,

$$
\begin{gathered}
\bar{m}=Z_{\mathrm{m}, \text { lat }}\left(g_{0}^{2}, a \mu\right) m_{\mathrm{q}}\left[1+a b_{m}\left(g_{0}^{2}\right) m_{\mathrm{q}}\right], \quad m_{\mathrm{q}}=m_{0}-m_{\mathrm{c}}, \\
b_{\mathrm{m}}=-0.5-0.07217(2) C_{F} g_{0}^{2}, \quad Z_{\mathrm{m}, \text { lat }}\left(g_{0}^{2}, a \mu\right)=1-\frac{1}{2 \pi^{2}} \log (a \mu) g_{0}^{2} .
\end{gathered}
$$

\subsection{Static approximation}

We can use the symmetries of the static theory to establish a relation between the renormalized, renormalization group invariant (RGI) axial vector and vector currents [2],

$$
\begin{gathered}
V_{0}^{\mathrm{HQET}}=C_{\mathrm{PS}}\left(M_{b} / \Lambda_{\overline{M S}}\right) Z_{\mathrm{A}, \mathrm{RGI}}^{\text {stat }}\left(g_{0}\right) Z_{\mathrm{V} / \mathrm{A}}^{\text {stat }}\left(g_{0}\right) V_{0}^{\text {stat }}, \\
A_{k}^{\mathrm{HQET}}=C_{\mathrm{V}}\left(M_{b} / \Lambda_{\overline{M S}}\right) Z_{\mathrm{A}, \mathrm{RGI}}^{\text {stat }}\left(g_{0}\right) Z_{\mathrm{V} / \mathrm{A}}^{\text {stat }}\left(g_{0}\right) A_{k}^{\text {stat }} .
\end{gathered}
$$

At one loop level, we have in the lattice minimal subtraction scheme

$$
\begin{array}{r}
\left(V_{\text {lat }}^{\text {stat }}\right)_{0}(\mu)=Z_{\mathrm{A}, \text { lat }}^{\text {stat }}(\mu) Z_{\mathrm{V} / \mathrm{A}}^{\text {stat }} V_{0}^{\text {stat }}, \quad\left(A_{\text {lat }}^{\text {stat }}\right)_{k}(\mu)=Z_{\mathrm{A}, \text { lat }}^{\text {stat }}(\mu) Z_{\mathrm{V} / \mathrm{A}}^{\text {stat }} A_{k}^{\text {stat }}, \\
Z_{\mathrm{A}, \text { lat }}^{\text {stat }}(\mu)=1-\gamma_{0} \log (a \mu) g_{0}^{2}+O\left(g_{0}^{4}\right), \quad Z_{\mathrm{V} / \mathrm{A}}^{\text {stat }}=1+\left(Z_{\mathrm{V} / \mathrm{A}}^{\text {stat }}\right)^{(1)} g_{0}^{2}+O\left(g_{0}^{4}\right),
\end{array}
$$

with (c.f. [18-20])

$$
\left(Z_{\mathrm{V} / \mathrm{A}}^{\mathrm{stat}}\right)^{(1)}=0.0521(1), \quad \gamma_{0}=-\frac{1}{4 \pi^{2}} .
$$

We define the static counterparts to $\Phi^{\mathrm{A}_{k}}$ and $\Phi^{\mathrm{V}_{0}}$,

$$
X_{\mathrm{A}_{k}}(\mu)=\log \left(Z_{\mathrm{A}, \text { lat }}^{\text {stat }}(\mu) Z_{\mathrm{V} / \mathrm{A}}^{\text {stat }}\right)+X_{\mathrm{A}_{k}}^{\text {bare }}, \quad X_{\mathrm{V}_{0}}(\mu)=\log \left(Z_{\mathrm{A}, \text { lat }}^{\text {stat }}(\mu) Z_{\mathrm{V} / \mathrm{A}}^{\text {stat }}\right)+X_{\mathrm{V}_{0}}^{\text {bare }},
$$

with $X_{\mathrm{A}_{k}}^{\text {bare }}$ and $X_{\mathrm{V}_{0}}^{\text {bare }}$ given (without the renormalization factors) as in (2.3) with the heavy quark flavor replaced by a static one. We may then expect that at one loop level

$$
\Phi^{\mathrm{V}_{0}}(L, \bar{m})=B_{\mathrm{A}}^{\text {stat }} g_{0}^{2}+X_{\mathrm{V}}(\bar{m})+O(1 / \bar{m}), \quad \Phi^{\mathrm{A}_{k}}(L, \bar{m})=B_{\mathrm{V}}^{\text {stat }} g_{0}^{2}+X_{\mathrm{A}}(\bar{m})+O(1 / \bar{m}),
$$

where we have from [2], using the ratio of $C_{\mathrm{V}}$ and $C_{\mathrm{PS}}$,

$$
B_{\mathrm{A}}^{\text {stat }}=-0.137(1), \quad B_{\mathrm{A}}^{\text {stat }}-B_{\mathrm{V}}^{\text {stat }}=0.016900 .
$$




\section{Results}

\subsection{Tree level}

Tree level results for $\Phi^{\mathrm{V}_{0}}$ and $\Phi^{\mathrm{A}_{k}}$ are shown in figure 1 , the points at $1 / z=0$ correspond to the static values $X_{\mathrm{A}_{k}}^{(0)}$ and $X_{\mathrm{V}_{0}}^{(0)}$. For $\theta=0.5,1.0$, the dependence on $1 / z$ is linear with a small slope. In the interesting region of $z=10$, which is the typical matching point for B physics used in [21], the $1 / z$ corrections at tree level are of a few percent. For $\theta=0$, there is no dependence on $z$ and $L / a$ at tree level for both observables. The continuum limit at tree level was extracted from computations with $L / a$ up to 200, using the fitting procedure explained in [22].

\subsection{One loop}

The observables $f_{1}, \Phi^{\mathrm{V}_{0}}$, and $\Phi^{\mathrm{A}_{k}}$ were calculated at one loop level for $z=4,6,8,10$ (and at $z=0$ for $f_{1}, k_{1}$ ), with lattice resolutions up to $L / a=40$, and for $\theta \in\{0.0,0.5,1.0\}$. Furthermore, we evaluated the static counterparts $f_{1}^{\text {stat }}, \Phi^{\mathrm{V}_{0} \text {,stat }}$ and $\Phi^{\mathrm{A}_{k} \text {,stat }}$ for lattices with $L / a$ up to 28 . No bigger lattice sizes are required for the HQET quantities, since their continuum limit is easier to obtain due to a weaker $a / L$-dependence. The continuum values are presented in figure 2 , the points for $1 / z=0$ are again the static values on which we will comment on shortly. The extraction of the continuum values is done with the method mentioned above. To establish the connection to the static limit, we define the one loop quantities

$$
\begin{aligned}
G_{\alpha}^{(1)}(z) & =\Phi^{\alpha,(1)}(z)+\gamma_{0} \log (z) \stackrel{1 / z \rightarrow 0}{\longrightarrow} H_{\alpha}^{(1)}, \quad \alpha \in\left\{V_{0}, A_{1}\right\}, \\
H_{\mathrm{V}_{0}}^{(1)} & =X_{\mathrm{V}_{0}}^{\text {bare, }(1)}+\left(Z_{\mathrm{V} / \mathrm{A}}^{\text {stat }}\right)^{(1)}+B_{\mathrm{A}}^{\text {stat }}-\gamma_{0} \log (a / L), \\
H_{\mathrm{A}_{k}}^{(1)} & =X_{\mathrm{A}_{k}}^{\text {bare, }(1)}+\left(Z_{\mathrm{V} / \mathrm{A}}^{\text {stat }}\right)^{(1)}+B_{\mathrm{V}}^{\text {stat }}-\gamma_{0} \log (a / L) .
\end{aligned}
$$

The fit functions included in the plots are of the form $G_{\alpha}^{(1)}(z)=H_{\alpha}^{(1)}+c_{1}^{\alpha} / z+c_{2}^{\alpha} / z^{2}$. Even though they are only indicative (since possible terms of the form $\log (z) / z^{n}$ are not included), one can anticipate that the higher order corrections corresponding to $c_{2}$ are rather small. We did not attempt to fit the logarithmic terms due to the small number of available data points.

In the case of $\Phi^{\mathrm{A}_{k}}$, a value of $\theta=0$ seems to minimize the higher order corrections in $1 / z$ both at tree level and at one loop. For $\Phi^{\mathrm{V}_{0}}$, the value $\theta=0.5$ seems to be a good compromise considering the corrections at the two orders in perturbation theory we investigated. One should always keep in mind that the small observed dependence on $1 / z$ will be eliminated once the $1 / m_{\mathrm{h}}$ corrections are included in the effective theory. Finally only the $1 / z^{2}$-terms, manifesting themselves in the curvature of the fits, will remain as corrections.

\section{Conclusions}

We have seen that the proposed observables for the matching are dominated by the lowest orders in $1 / m$ and hence one would strongly suggest to use them in a non-perturbative study. For the phase angle $\theta$, we would suggest the value 0.5 as a compromise to minimize $1 / z$-effects at one-loop and tree level, but the current study does not indicate a strong preference for this value. 
A further investigation including the matching conditions for all 19 parameters is currently carried out within the ALPHA collaboration.

We could also demonstrate the usefulness of the pastor software package for applications beyond the classical realms of lattice perturbation theory such as renormalization and improvement. The publication of an in-depth description of pastor along with its source code is planned for the near future.

\section{Acknowledgments}

The calculations were carried out on the PC farm at DESY Zeuthen. This work has been supported by the DFG Sonderforschungsbereich TR9 Computergestützte Theoretische Teilchenphysik and the Research Executive Agency (REA) of the European Union under Grant Agreement number PITN-GA-2009-238353 (ITN STRONGnet).

\section{References}

[1] D. Hesse and R. Sommer, to be published, arXiv:1211.0866 [hep-lat].

[2] R. Sommer, arXiv:1008.0710 [hep-lat].

[3] J. Heitger et al. [ALPHA Collaboration], JHEP 0402, 022 (2004) [hep-lat/0310035].

[4] B. Blossier, M. della Morte, N. Garron and R. Sommer, JHEP 1006, 002 (2010) [arXiv:1001.4783 [hep-lat]].

[5] B. Blossier et al. [ALPHA Collaboration], JHEP 1005, 074 (2010) [arXiv:1004.2661 [hep-lat]].

[6] B. Blossier et al. [ALPHA Collaboration], JHEP 1012, 039 (2010) [arXiv:1006.5816 [hep-lat]].

[7] M. Della Morte, P. Fritzsch and J. Heitger, JHEP 0702, 079 (2007) [hep-lat/0611036].

[8] B. Blossier, M. Della Morte, P. Fritzsch, N. Garron, J. Heitger, H. Simma, R. Sommer and N. Tantalo, JHEP 1209, 132 (2012) [arXiv:1203.6516 [hep-lat]].

[9] D. Hesse, R. Sommer and G. von Hippel, PoS LATTICE 2011, 229 (2011).

[10] M. Lüscher, R. Narayanan, P. Weisz and U. Wolff, Nucl. Phys. B 384, 168 (1992) [hep-lat/9207009].

[11] S. Sint, Nucl. Phys. B 421, 135 (1994) [hep-lat/9312079].

[12] M. Lüscher, S. Sint, R. Sommer and P. Weisz, Nucl. Phys. B 478, 365 (1996) [hep-lat/9605038].

[13] J. Heitger et al. [ALPHA Collaboration], JHEP 0411 (2004) 048 [hep-ph/0407227].

[14] M. Lüscher, S. Sint, R. Sommer and H. Wittig, Nucl. Phys. B 491, 344 (1997) [hep-lat/9611015].

[15] L. Maiani and G. Martinelli, Phys. Lett. B 178, 265 (1986).

[16] S. Sint and P. Weisz, Nucl. Phys. B 502, 251 (1997) [hep-lat/9704001].

[17] E. Gabrielli, G. Martinelli, C. Pittori, G. Heatlie and C. T. Sachrajda, Nucl. Phys. B 362, 475 (1991).

[18] F. Palombi, JHEP 0801, 021 (2008) [arXiv:0706.2460 [hep-lat]].

[19] M. A. Shifman and M. B. Voloshin, Sov. J. Nucl. Phys. 45, 292 (1987) [Yad. Fiz. 45, 463 (1987)].

[20] H. D. Politzer and M. B. Wise, Phys. Lett. B 206, 681 (1988).

[21] M. Della Morte, N. Garron, M. Papinutto and R. Sommer, JHEP 0701, 007 (2007) [hep-ph/0609294].

[22] A. Bode et al. [ALPHA Collaboration], Nucl. Phys. B 576, 517 (2000) [Erratum-ibid. B 600, 453 (2001)] [Erratum-ibid. B 608, 481 (2001)] [hep-lat/9911018]. 


\section{A. Plots}
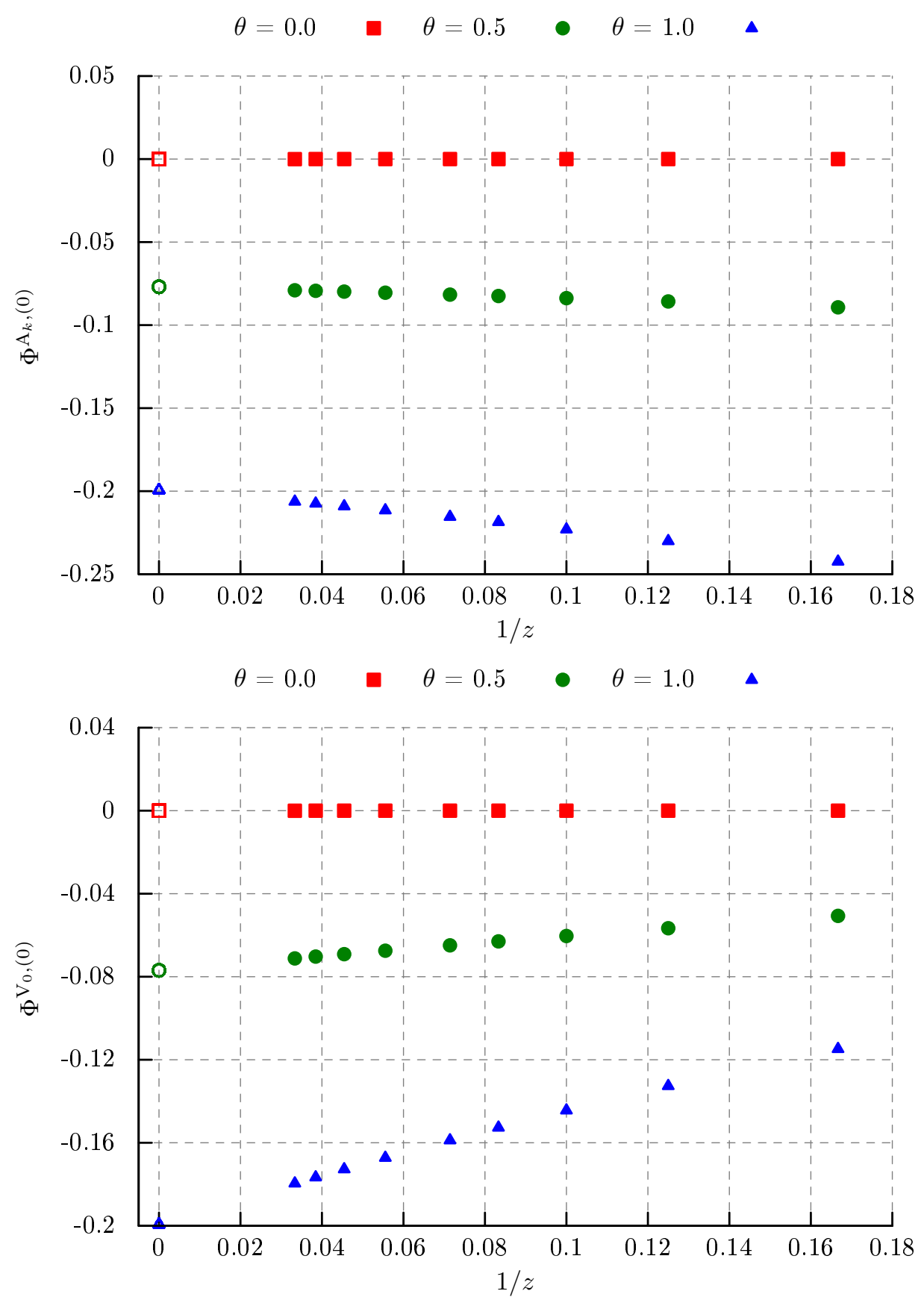

Figure 1: $\Phi^{\mathrm{A}_{k}}$ and $\Phi^{\mathrm{V}_{0}}$ at tree level in the continuum limit. The point size is bigger than the error. 

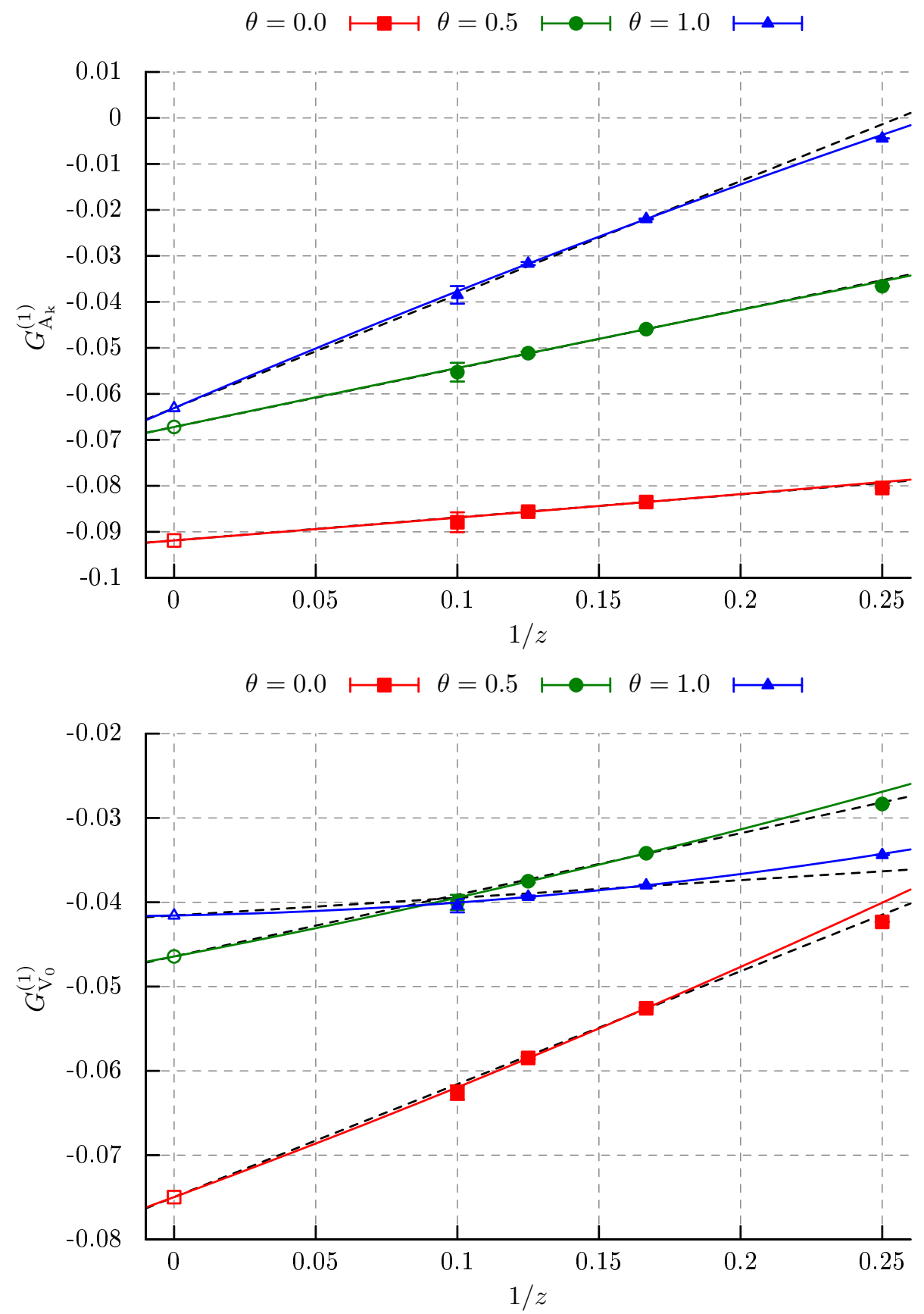

Figure 2: $G_{\mathrm{A}_{k}}^{(1)}$ and $G_{\mathrm{V}_{0}}^{(1)}$ in the continuum limit. The dashed lines are a linear fit in $1 / z$, setting $c_{2}=0$. The data points at $z=4$ are not included in the fits. 